\title{
Form Finding of Sparse Structures with Continuum Topology Optimization
}

\author{
Salam Rahmatalla ${ }^{1}$ and Colby C. Swan, M.ASCE ${ }^{2}$
}

\begin{abstract}
A continuum topology optimization methodology suitable for finding optimal forms of large-scale sparse structures is presented. Since the need to avoid long compressive spans can be critical in determining the optimal form of such structures, a formulation is used wherein the structure is modeled as a linear elastic continuum subjected to design loads, and optimized in form to maximize the minimum critical buckling load. Numerical issues pertinent to accurate solution of the linearized buckling eigenvalue problem and accurate design sensitivity analysis are discussed. The performance of the proposed design formulation is demonstrated on a few problems designed to find optimal forms of a canyon bridge, long-span bridges, and an electrical transmission tower. In all cases, very credible structural forms are obtained with the proposed design formulation. The results of the design examples solved are typically superior structural forms with regard to buckling stability than those obtained to minimize the mean structural compliance.
\end{abstract}

DOI: 10.1061/(ASCE)0733-9445(2003)129:12(1707)

CE Database subject headings: Optimization; Buckling; Sensitivity analysis; Eigenvalues.

\section{Introduction}

Designing sparse large-scale structures that are not susceptible to buckling instabilities is a longstanding challenge in structural design optimization. Many structural design optimization methods are in use today and include cross-section selection, or size and shape optimization of structural cross sections. These types of methods are applicable toward the final stages of designing a structure, but they tend not to be very helpful when one is trying to find the optimal forms of a structure based on its size (or span), support conditions, and the loads that it is expected to carry. While the designer's intuition and awareness of design precedents will always play a vital role, structural topology optimization methods are objective tools that might also be very useful in identifying suitable, if not optimal, structural forms. Among structural topology optimization frameworks are there are essentially two classes of methods (see Ohsaki and Swan 2002): (1) discrete ground-structure topology optimization methods and (2) continuum structural topology optimization methods.

One of the pioneering works in the field of structural topology optimization per se involved presentation of optimal discrete truss structures now called Michell structures (Michell 1904). More recently, discrete ground structure truss topology optimization methods have been employed (Dorn et al. 1964; Dobbs and Felton 1969; Hemp 1973; Rozvany 1976; Save and Prager 1990) and

${ }^{1}$ Dept. of Civil and Environmental Engineering, Center for ComputerAided Design, The Univ. of Iowa, Iowa City, IA 52242.

${ }^{2}$ Dept. of Civil and Environmental Engineering, Center for ComputerAided Design, The Univ. of Iowa, Iowa City, IA 52242 (corresponding author). E-mail: colby-swan@uiowa.edu

Note. Associate Editor: Shahram Pezeshk. Discussion open until May 1, 2004. Separate discussions must be submitted for individual papers. To extend the closing date by one month, a written request must be filed with the ASCE Managing Editor. The manuscript for this paper was submitted for review and possible publication on July 18, 2002; approved on November 19, 2002. This paper is part of the Journal of Structural Engineering, Vol. 129, No. 12, December 1, 2003. (CASCE, ISSN 0733-9445/ 2003/12-1707-1716/\$18.00. they are now quite standard. In the recent past, such methods have been investigated quite extensively for civil engineering type structures with a good deal of attention paid to achievement of designs that are stable with respect to design loads (Oberndorfer et al. 1996; Rozvany 1996; Zou 1996; Achtziger 1999; Bojczuk and Mróz 1999; Kočvara 2002). In such frameworks, an attempt is typically made to achieve stable designs by enforcing local Euler buckling constraints. This can be challenging, due to difficulty in identifying buckling lengths that can be considerably larger than individual member lengths when several collinear and compressive members form an isolated sequence.

While discrete structural optimization methods date back at least one century to the work of Michell, continuum structural topology optimization methods have more recent origins, developed in the 1980s as extensions of shape and size optimization techniques (Cheng and Olhoff 1981; Bendsøe and Kikuchi 1988). In these methods, the structure is modeled as a continuum and the form of the structural system is optimized using a system of distributed continuous design parameters [see the recent review by Eschenauer and Olhoff (2001) for a survey of the numerous continuum topology optimization formulations developed over the past 2 decades]. Since these methods are not in any way restricted to truss-like structures, they have been investigated for a considerable range of applications including: the design of composite material microstructures, (see Swan and Arora 1997 for one example); compliant mechanisms in microelectromechanical systems (Yin and Ananthasuresh 2002) intermediate scale plate and shell structures (Swan and Kosaka 1997); and civil engineering type structures (Mijar et al. 1998; Swan et al. 1998).

In spite of its arguable successes in design of small and intermediate scale mechanical systems, there have been some challenges in applying continuum topology optimization to the conceptual design of large-scale civil engineering type structures for which economy of material is typically a vital issue. Large-scale civil engineering structures such as bridges and transmission towers are characteristically very sparse with the volume of material that comprises the structural system constituting a very small fraction $(\leqslant 5 \%)$ of the structure's total envelope volume. Con- 
tinuum topology optimization methods, unlike discrete truss optimization methods, have some difficulty modeling this sparsity since it requires extremely refined models with very high degree of freedom counts. If the design method cannot capture this sparsity, then it will have difficulty replicating and addressing the vibrational and stability characteristics of the structure. As a result, when continuum topology methods were applied in preceding efforts to design the form of structures for both optimal stiffness and vibrational characteristics, the designed forms of the structure were realistic, but the computed vibrational characteristics, such as eigenfrequencies, were not (Ma et al. 1995; Mijar et al. 1998; Swan et al. 1998; Min et al. 2000).

For large-scale sparse structures, stability considerations can often be the controlling factor in determining the overall form of the structure. For example, in the design of long-span bridges, tension structures are typically optimal since they preclude potential buckling under the traffic loading and self-weight loading. Previous applications of continuum topology optimization to finding optimal forms of bridges based on minimization of the linear elastic structural compliance under traffic loading, or maximization of the fundamental vibrational eigenfrequencies of the structure, or combinations of both functionals using multicriterion optimization approaches (Swan et al. 1998) have not been satisfactory. Neither linear elastic compliance, nor vibrational eigenfrequencies of a structural system are directly related to structural stability in sparse structures. For this reason, the resulting concept designs of structures from these preceding works just cited often feature long slender compression members that would be highly problematic if the design were pursued from the conceptual stage through the detailed design stage.

In continuum structural topology optimization formulations, achievement of designs that are inherently stable in relation to the design loads can be very challenging. For example, it can be difficult to preclude local buckling behaviors with Euler-type constraints, since it is very difficult to identify discrete structural members, their geometrical properties, and their end support conditions from the vector of design variables. One promising approach to addressing global geometrical instabilities in continuum structural topology optimization (Guedes and Rodrigues 1995; Neves et al. 1995) and that has been employed in design of bracing systems for portal frames is to model the structure as a linearly elastic system and to use the minimum critical buckling load computed via eigenvalue analysis either in the objective function or as a design constraint. While such an approach is very straightforward in principle, a complicating factor is that when the buckling eigenvalues of the structure are nonsimple (repeated), their design derivatives are discontinuous. Accordingly, an alternative approach to designing stable, sparse structures in a continuum topology framework is to model the structure as an elastic continuum taking into account finite deformation effects and the associated instabilities (Buhl et al. 2000; Bruns and Tortorelli 2001; Gea and Luo 2001; Rahmatalla and Swan 2003). Nevertheless, the advantage of the first approach over the latter is its substantially lower computational cost.

In the body of this paper, a linear elastic continuum topology formulation is introduced for minimization of mean structural compliance, and for maximization of minimum critical buckling values computed by linearized buckling analysis. One of the intents is to demonstrate the advantages of designing to maximize minimum critical buckling loads as opposed to designing to minimize linear elastic structural compliance. This point is salient as designing for compliance minimization has been frequently explored in the research literature, and yet it will generally not lead to satisfactory concept designs of large-scale sparse structures.

In this paper, basic elements of continuum structural topology optimization are presented including material distribution design parameters, problem statements, and design sensitivity analysis expressions. Finally, practical examples including concept designs of large structures are demonstrated followed by discussion and concluding remarks.

\section{Problem Formulations}

\section{Structural Model and Material Layout Description}

The objective of continuum structural optimization is to find a layout of a structural material of specified properties in a defined spatial region that provides optimum structural performance. In order that the widest possible class of structural layouts can be considered, the methods in question must accommodate such generality. In this work, the spatial region that the candidate structural models can occupy is denoted $\Omega_{s}$. To facilitate both description of the structural material layout in $\Omega_{s}$ and analysis of the performance associated with each layout considered, the domain is discretized into a relatively fine mesh of nodes and finite elements.

It is desired that at the end of the form-finding process, the structural region $\Omega_{s}$ will be decomposed into a collection of regions cumulatively denoted $\Omega_{A}$ that contain the structural material in question, and the remaining regions $\Omega_{B}=\Omega_{s} / \Omega_{A}$ that are devoid of structural material. Since solution of the form-finding problem in this way is ill-posed, an alternative relaxed approach is usually employed, wherein it is assumed that an amorphous "mixture" of structural material A and a void material B exists throughout the structural region $\Omega_{s}$. In each region of $\Omega_{s}$, the nature of the mixture is characterized by a local volumetric density $\phi_{A}$ of structural material A. By permitting mixtures, the structural material A and a fictitious void material B are allowed to simultaneously occupy an infinitesimal neighborhood about each Lagrangian point $\mathbf{X} \in \Omega_{s}$. The volumetric density of structural material A at a fixed Lagrangian point $\mathbf{X} \in \Omega_{s}$ is denoted by $\phi_{A}(\mathbf{X})$ and represents the fraction of an infinitesimal region surrounding point $\mathbf{X}$ occupied by material A. Natural constraints upon the volumetric densities are

$$
0 \leqslant \phi_{A}(X) \leqslant 1 ; \quad 0 \leqslant \phi_{B}(X) \leqslant 1 ; \quad \phi_{A}(X)+\phi_{B}(X)=1
$$

Clearly, when $\phi_{A}(\mathbf{X})=1$ the point $\mathbf{X}$ contains solid structural material, and when $\phi_{A}(\mathbf{X})=0$ the point $\mathbf{X}$ is devoid of structural material. The last physical constraint of Eq. (1) states that the material volume fractions at $\mathbf{X}$ are not independent and so one need only be concerned with the layout of structural material A. The design of a structure is here considered to be the spatial distribution of the structural material A in $\Omega_{s}$.

To describe the distribution of material A throughout $\Omega_{s}$ using a finite number of design parameters, the volumetric density at each of the NUMNP nodal point forms a set of $N U M N P$ design variables. These are then interpolated over the space of all intermediate points in the structure using the nodal shape functions

$$
\phi(\mathbf{X})=\sum_{i=1}^{N U M N P} \mathrm{~b}_{i} N_{i}(\mathbf{X}) \quad \forall \mathbf{X} \in \Omega_{s}
$$

where $\mathrm{b}_{i}=$ nodal volumetric density values associated with the structural material; and $N_{i}(\mathbf{X})=$ nodal shape functions. This approach yields a $C^{0}$ continuous design variable field that is not susceptible to "checkerboarding" instabilities. 
Given the finite element model of the structural region $\Omega_{s}$, the structural loads and restraints (or supports) on this region are specified as the set of design loads. For each set of design loadings, and for each realization of the design vector $\mathbf{b}$ $=\left\{b_{1}, b_{2}, \ldots, b_{N U M N P}\right\}$, the response performance of the structure will be analyzed as a boundary value problem. From the computed response of the structure, the performance of the structure will be quantified, as will be the sensitivity of the performance to variations in the design variables.

\section{Constitutive Mixing Rules}

In the proposed design framework, each finite element comprising the spatial domain $\Omega_{s}$ of the structure will generally contain a spatially varying mixture of the structural and void materials. It is necessary to prescribe the stiffness (or elastic moduli) of such mixtures in terms of the stiffness characteristics of the solid material $\mathbf{C}_{\text {solid }}$, those of the fictitious void material $\mathbf{C}_{\text {void }}$, and the local volumetric density of the structural material $\phi(\mathbf{X})$. Here, the well-known powerlaw formula (Bendsøe and Sigmund 1999) is used to accomplish this task, providing the local effective stiffness of the mixture $\mathbf{C}^{*}$ as

$$
\mathbf{C}^{*}=\phi^{p} \mathbf{C}_{\text {solid }}+\left(1-\phi^{p}\right) \mathbf{C}_{\text {void }}
$$

where typically the mixing rule parameter $p \in[1,4]$. With $p=1$, the Voigt rule of mixtures is obtained which does not penalize mixtures, but which does yield a convex formulation for compliance minimization problems (Swan and Kosaka 1997) so that only one solution exists for the design problem. With $p=4$, mixtures are penalized in the final design, so that regions of $\Omega_{s}$ tend to be either solid or void, but the optimization problem is not convex, and will admit a number of solutions that satisfy the first order optimality conditions.

\section{Structural Analysis}

For each design, a structural analysis problem is solved on the continuum domain $\Omega_{s}$. In general terms, the structural analysis problem solved for each realization of the design vector $\mathbf{b}$ is the following: Find the displacement field $\mathbf{u}(\mathbf{X}) \Omega_{s} \rightarrow \mathcal{R}^{3}$ such that the variational equilibrium problem is solved

$$
\int_{\Omega_{s}} \boldsymbol{\sigma}: \delta \in \mathrm{d} \Omega_{s}=\int_{\Gamma_{\mathrm{s}}} \mathbf{h} \cdot \delta \mathbf{u} d \Gamma_{\mathrm{s}}+\int_{\Omega_{s}} \rho \mathbf{g} \cdot \delta \mathbf{u} d \Omega_{s}
$$

where $\sigma(\mathbf{X})=$ local stress field in the structure; $\mathbf{h}=$ traction vector consistent with the design loads being applied to the structure; $\rho(\mathbf{X})=$ local mass density of the structural material; $\mathbf{g}=$ gravitational body force vector; $\delta \mathbf{u}=$ kinematically admissible variational displacement field; and $\delta \boldsymbol{\epsilon}=$ corresponding variational strain field. In the structural model, the material features linear elastic behavior such that $\boldsymbol{\sigma}=\mathbf{C}^{*}: \boldsymbol{\epsilon}$, where the effective elasticity tensor is design dependent and prescribed in accordance with Eq. (3). The matrix problem associated with variational equilibrium of the discrete finite element structural model, for which $\mathbf{u}(\mathbf{X})$ $=\sum_{i} N_{i}(\mathbf{X}) \mathbf{u}_{i}$ is

$$
\mathbf{0}=\mathbf{K} \cdot \mathbf{u}-\mathbf{f}^{\text {ext }}=\mathbf{f}^{\text {int }}-\mathbf{f}^{\text {ext }}
$$

where

$$
\begin{aligned}
K_{j k}^{L M} & =\int_{\Omega_{s}} B_{m j}^{L} C_{m n}^{*} B_{n k}^{M} d \Omega_{s} \\
\mathbf{f}^{\mathrm{int}} & =\mathbf{K} \cdot \mathbf{u}=\int_{\Omega_{s}} \mathbf{B}^{T} \boldsymbol{\sigma} d \Omega_{s}
\end{aligned}
$$

$$
\mathbf{f}^{\mathrm{ext}}=\int_{\Gamma_{s}} N \mathbf{h} d \Gamma_{s}+\int_{\Omega_{s}} N \rho \mathbf{g} d \Omega_{s}
$$

In all of the above, $N$ denotes the nodal shape functions and $\mathbf{B}$ denotes the standard strain-displacement matrices (cf. Bathe 1996). The structural stiffness matrix $\mathbf{K}$ is positive definite due to the characteristics of the effective elasticity tensor $\mathbf{C}^{*}$, and this guarantees a unique solution to the structural analysis problem for each realization of the design $\mathbf{b}$.

Once the equilibrium solution to the problem of Eq. (5) is obtained, then the linearized geometrical stiffness matrix $\mathbf{G}$ can be computed based on the stress field $\sigma$ in the structure

$$
G_{j k}^{L M}=\int_{\Omega_{s}} N_{, m}^{L} N_{, n}^{M} \sigma_{\mathrm{mn}} \delta_{j k} d \Omega_{s}
$$

It is worth noting that $\mathbf{G}$ is not necessarily positive definite but rather depends heavily upon the nature of the stress field in the structure. A purely tensile stress field clearly makes $\mathbf{G}$ positive definite, although for any compressive stresses, $\mathbf{G}$ will not be positive definite.

\section{Structural Performance Measures}

\section{Overview}

As noted previously, structural topology design problems can be formulated in a number of alternative ways through utilization of assorted objective and constraint functions. Generally, the objective function measures the performance of the structure, and the constraint function limits the amount of structural material that can be used, although the roles can be reversed equally well. The significant aspects of using CSTO to design large-scale sparse structures can be demonstrated here using the linear elastic structural compliance performance measure and the critical load buckling factor.

\section{Linear Elastic Structural Compliance}

If a structure features a linear elastic response behavior, the resulting displacement field $\mathbf{u}$ in response to a set of applied external loads $\mathbf{f}^{\text {ext }}$ will be simply $\mathbf{u}=\mathbf{K}^{-1} \cdot \mathbf{f}^{\text {ext }}$, where $\mathbf{K}$ represents the stiffness matrix of the structure. For a given set of loads, the compliance $\Pi(\mathbf{b})$ of the structure is simply

$$
\Pi(\mathbf{b})=\frac{1}{2} \mathbf{f}^{\mathrm{ext}} \cdot \mathbf{u}
$$

Structural concept designs $\mathbf{b}$ that are stiff with respect to the applied loads will have small compliance $\Pi(\mathbf{b})$, whereas structures that are not stiff with respect to the applied loads will have large compliance. To facilitate usage of gradient-based optimization solution techniques, it is necessary to compute the design derivatives of the compliance function. It can be shown that the design gradient of structural compliance is provided by the following expression:

$$
\frac{d \Pi}{d \mathbf{b}}=-\frac{1}{2} \mathbf{u} \cdot\left(\frac{\partial \mathbf{K}}{\partial \mathbf{b}} \cdot \mathbf{u}-\frac{\partial \mathbf{f}^{\mathrm{ext}}}{\partial \mathbf{b}}\right)
$$

\section{Linearized Bucking Performance Measure}

Linearized buckling eigenvalue analysis proceeds as follows: A prescribed force loading $\mathbf{f}^{\text {ext }}$ is applied to the structure with its magnitude necessarily being less than that required to induce geometric instability in the structure. Once the resulting linear, elas- 
tostatic displacement solution $\mathbf{u}=\left\{u_{i}\right\} \in \mathbf{R}^{N}$ in response to the applied loading $\mathbf{f}^{\text {ext }}$ is obtained $\left(\mathbf{K} \cdot \mathbf{u}=\mathbf{f}^{\text {ext }}\right)$, where $\mathbf{K}$ is the linearized stiffness matrix, then the following eigenvalue problem is solved:

$$
[\mathbf{K}(\mathbf{b})+\lambda \mathbf{G}(\mathbf{u}, \mathbf{b})] \cdot \boldsymbol{\psi}=\mathbf{0}
$$

In the preceding, $\mathbf{b}=\left\{\mathbf{b}_{e}\right\} \in \mathbf{R}^{M}$ is again the vector of design variables; $\mathbf{K}=$ tangent stiffness operator; $\mathbf{G}(\mathbf{u}, \mathbf{b})=$ linearized geometric stiffness matrix; $\lambda=-(\boldsymbol{\psi} \cdot \mathbf{K} \cdot \boldsymbol{\psi} / \boldsymbol{\psi} \cdot \mathbf{G} \cdot \boldsymbol{\psi})=$ eigenvalue denoting the magnitude by which $\mathbf{f}^{\text {ext }}$ must be scaled to create instability in the structure; and $\boldsymbol{\psi}=$ normalized eigenvector satisfying $\boldsymbol{\psi} \cdot \mathbf{K} \cdot \boldsymbol{\psi}=1$. To avoid numerical difficulties in the solution of Eq. (10) stemming from the indefinite characteristics of $\mathbf{G}$, it is common (Bathe 1996) to solve a modified eigenvalue problem that deals with two positive definite matrices

$$
[(\mathbf{K}+\mathbf{G})-\gamma \mathbf{K}] \cdot \boldsymbol{\psi}=\mathbf{0}
$$

where

$$
\gamma=\frac{\lambda-1}{\lambda} \Leftrightarrow \lambda=\frac{1}{1-\gamma}
$$

In Eq. (11), the matrix $\mathbf{K}$ is positive definite irrespective of the loading applied to the structure, whereas the matrix $(\mathbf{K}+\mathbf{G})$ will only be positive definite when the magnitude of the loading applied to the structural model is less than the critical magnitude that creates instability in accordance with linearized buckling theory.

The design problem is formulated to maximize the calculated minimum-buckling load factor $(\lambda)$, and accordingly the objective function $f_{E}$ to be minimized for this problem would simply be the reciprocal of the lowest eigenvalue $\lambda$ as follows:

$$
f_{E}(\mathbf{u}, \mathbf{b})=\frac{1}{\min (\lambda)}
$$

The optimization problem is thus stated to minimize the reciprocal of the first (or minimum) critical buckling load as follows:

$$
\min _{\mathbf{b}, \mathbf{u}} f_{E}=\min _{\mathbf{b}, \mathbf{u}}\left(\frac{1}{\lambda}\right)=\min _{\mathbf{b}, \mathbf{u}}\left(-\max _{\|\psi\| \neq 0} \frac{\boldsymbol{\psi} \cdot \mathbf{G} \cdot \boldsymbol{\psi}}{\boldsymbol{\psi} \cdot \mathbf{K} \cdot \boldsymbol{\psi}}\right)
$$

subject to the normal bound constraints on the design variables Eq. (1), the linear structural equilibrium state Eq. (5), and a constraint on material resources.

The design gradient of the objective function can be expressed as

$$
\frac{d f_{E}}{d \mathbf{b}}=\frac{\partial f_{E}}{\partial \mathbf{b}}+\frac{\partial f_{E}}{\partial \mathbf{u}} \cdot \frac{\partial \mathbf{u}}{\partial \mathbf{b}}
$$

To avoid explicit computation of the term $\partial \mathbf{u} / \partial \mathbf{b}$, adjoint design sensitivity analysis is employed by augmenting the objective function $f_{E}$ with the equilibrium state equation as follows:

$$
\Xi=f_{E}+\mathbf{u}^{a} \cdot \mathbf{r}
$$

where $\mathbf{u}^{a}=$ adjoint displacement vector which functions as a matrix of Lagrange multipliers and determined by the solution of a linear adjoint problem. The design derivative of the augmented Lagrangian is then written as follows:

$$
\frac{d \Xi}{d \mathbf{b}}=\left(\frac{\partial f_{E}}{\partial \mathbf{b}}+\mathbf{u}^{a} \cdot \frac{\partial \mathbf{r}}{\partial \mathbf{b}}\right)+\left[\frac{\partial f_{E}}{\partial \mathbf{u}}+\frac{\partial \mathbf{r}}{\partial \mathbf{u}} \cdot \mathbf{u}^{a}\right] \cdot \frac{\partial \mathbf{u}}{\partial \mathbf{b}}+\left(\mathbf{r} \cdot \frac{\partial \mathbf{u}^{a}}{\partial \mathbf{b}}\right)
$$

The last term of Eq. (17) vanishes due to satisfaction of the equi-

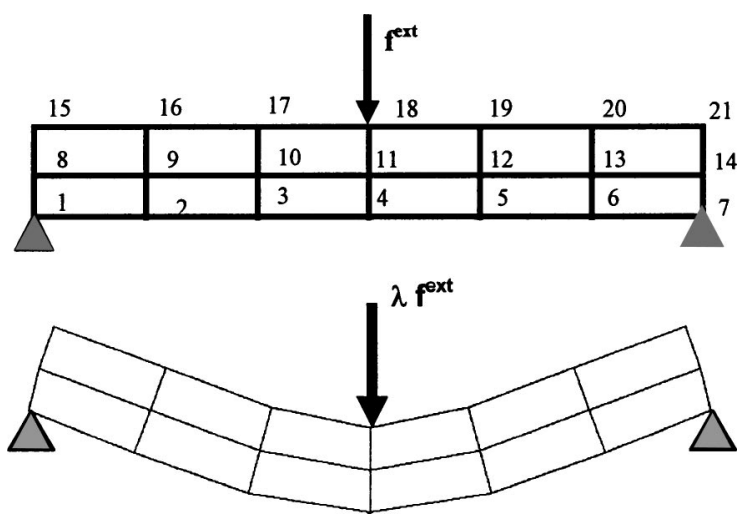

Fig. 1. Undeformed and deformed configurations of coarse beam model with first buckling mode shown

librium constraint $(\mathbf{r}=\mathbf{0})$, and the second term can be made to vanish by selecting the adjoint displacement vector to solve the following linear adjoint equality statement

$$
\mathbf{K} \cdot \mathbf{u}^{a}=\boldsymbol{\psi} \cdot \frac{\partial \mathbf{G}}{\partial \mathbf{u}} \cdot \boldsymbol{\psi}
$$

Since it can be shown that $d \Xi / d \mathbf{b}=d f_{E} / d \mathbf{b}$, it follows that the design gradient expression for the objective function is

$$
\frac{d f_{E}}{d \mathbf{b}}=-\boldsymbol{\psi} \cdot\left(\frac{\partial \mathbf{G}}{\partial \mathbf{b}}+\frac{1}{\lambda} \frac{\partial \mathbf{K}}{\partial \mathbf{b}}\right) \cdot \boldsymbol{\psi}+\mathbf{u}^{a} \cdot\left(\frac{\partial \mathbf{K}}{\partial \mathbf{b}} \cdot \mathbf{u}-\frac{\partial \mathbf{f}^{\mathrm{ext}}}{\partial \mathbf{b}}\right)
$$

The preceding expression is valid only when the minimum eigenvalue is a simple, or nonrepeated, eigenvalue. When the minimum eigenvalue is nonsimple, or repeated, the variation of the eigenvalue in design space is nonsmooth, and direct usage of the expression in Eq. (19) is technically incorrect (Choi et al. 1983; Seyranian et al. 1994). Resolution of this issue is nontrivial, although it can be ameliorated somewhat by using small and variable move limits in the design optimization process. Despite this challenge, designs that successfully maximize the buckling stability of a structural system can nevertheless be obtained.

\section{Numerical Issues}

Beyond the nonsmooth nature of repeated eigenvalues, when they occur, there are additional numerical issues associated with reliable solution of the buckling eigenvalue problem and the attendant effect on accurate sensitivity of buckling eigenvalues with respect to design changes. As noted previously, due to indefinite characteristics of the linearized $\mathbf{G}$, the modified buckling eigenvalue problem of Eq. (11) is usually solved rather than that of Eq. (10). However, when the magnitude of loading applied to the structure is greatly exceeded by that of $f^{\text {crit }}$, then $\lambda \rightarrow \infty$ and accordingly $\gamma \rightarrow 1$. As this happens, it is increasingly difficult to accurately compute both $\lambda$ and $d \lambda / d \mathbf{b}$. This is demonstrated by considering the simple beam model shown in Fig. 1 for which a load of magnitude $\left\|\mathbf{f}^{\text {ext }}\right\|=1 \mathrm{MN}$ is applied, and for which $f^{\text {crit }}$ $=\lambda *\left\|\mathbf{f}^{\text {ext }}\right\|=5.1855 \mathrm{MN}$, giving $\lambda=5.1855$. One would expect that as the magnitude of the loading applied to the structural model changed, the computed value of $\lambda$ would change in accor-

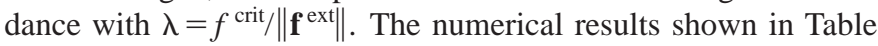
1 indicate otherwise, however, and that as the inaccuracy in computed values of $\lambda$ increases, the inaccuracy in the design sensitivities increases dramatically. Accordingly, when computing the 
Table 1. Buckling Factors Computed with Standard Methods for Model Shown in Fig. 1.

\begin{tabular}{ccccc}
\hline$f^{\text {ext }}$ & & & & Analytical DSA \\
$(\mathrm{N})$ & $\gamma$ & $\lambda$ & Node 14 & $\begin{array}{c}\text { Dinite difference } \\
\text { DSA }\end{array}$ \\
\hline $10^{2}$ & 0.999981 & $5.29781 \times 10^{4}$ & $-6.7096 \times 10^{-5}$ & $-6.7491 \times 10^{-5}$ \\
$10^{4}$ & 0.9981 & $5.24000 \times 10^{3}$ & $-6.8476 \times 10^{-3}$ & $-7.0158 \times 10^{-3}$ \\
$10^{6}$ & 0.8072 & $5.1855 \times 10^{0}$ & $-7.1877 \times 10^{-1}$ & $-7.1877 \times 10^{-1}$ \\
\hline
\end{tabular}

Note: Inaccuracies exist in computed eigenvalues and also in both analytical and finite difference design sensitivity results.

buckling eigenvalue of the structural model, greater accuracy is achieved by using a scaled load magnitude $\left\|\mathbf{f}^{\text {ext }}\right\|$ such that

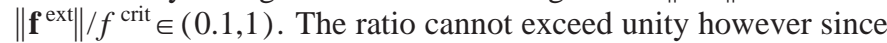
in that case $(\mathbf{K}+\mathbf{G})$ loses its positive definiteness. To avoid the problems associated with application of a load to the structural model that is either far too small (i.e. $\left\|\mathbf{f}^{\mathrm{ext}}\right\| / f^{\text {crit }} \ll 1$ ), or too large (i.e. $\left\|\mathbf{f}^{\text {ext }}\right\| / f^{\text {crit }} \geqslant 1$ ) an algorithm such as that shown in Fig. 2 is necessary.

The essential idea behind the proposed algorithm in Fig. 2 is that while a fixed set of structural loads, here denoted by $\mathbf{f}^{\text {ref }}$ are applied to the structural model for all realizations of the design vector $\mathbf{b}$, the computed value of $\lambda^{\text {ref }}=f^{\text {crit }}(\mathbf{b}) /\left\|\mathbf{f}^{\text {ref }}\right\|$ for each realization of the design $\mathbf{b}$ could either be excessively large (i.e., $\lambda^{\mathrm{ref}} \gg 1$ ) and thus contain a large amount of error, or alternatively too small (i.e., $\lambda^{\text {ref }} \leqslant 1$ ) making it very difficult to compute the minimum eigenvalue associated with Eq. (10). An accurate computation of the buckling eigenvalue with respect to the loads $\mathbf{f}^{\text {ref }}$ can be achieved, however, by iterative scaling of the actual system of loads applied to the structure $\mathbf{f}^{\text {ext }}=k^{*} \mathbf{f}^{\text {ref }}$ until $\lambda \in(1,10)$, where here $\lambda$ is the minimum eigenvalue with respect to the scaled loads. Since $f^{\text {crit }}(\mathbf{b})=\lambda^{\text {ref } *}\left\|\mathbf{f}^{\text {ref }}\right\|=\left(\lambda^{\text {ref }} / k\right)\left(k^{*}\left\|\mathbf{f}^{\text {ref }}\right\|\right)$ $=\lambda *\left\|\mathbf{f}^{\mathrm{ext}}\right\|$, it follows that $\lambda^{\mathrm{ref}}=k^{*} \lambda$. To demonstrate the effectiveness of the proposed algorithm, the computed buckling eigenvalues and their design gradients associated with the test problem of Fig. 1 were computed using the algorithm of Fig. 2, and the

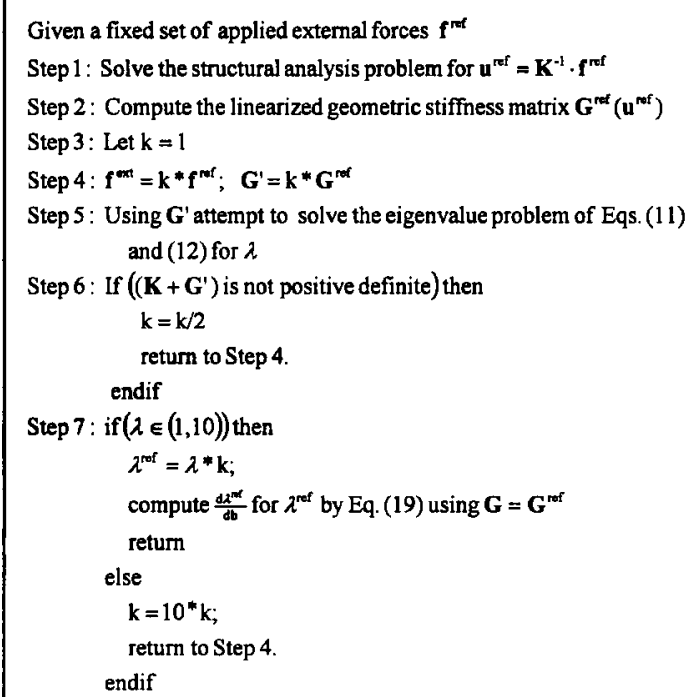

Fig. 2. Algorithm for accurate computation of buckling load factor eigenvalue $\lambda^{\text {ref }}$ with respect to fixed set of design loads $f^{\text {ref }}$ results presented in Table 2 . As can be seen from this table, the computed buckling eigenvalues now scale in inverse proportion to the magnitude of the applied loads $\mathbf{f}^{\text {ref }}$, and the computed design sensitivity results are now in close agreement with those computed by converged finite difference analysis.

\section{Demonstrative Examples}

\section{Material Properties and Mixing Rules}

In the following examples, the CSTO formulation to maximize minimum critical buckling loads computed by linearized eigenvalue analysis is tested and compared with the more commonly used compliance minimization formulation. In all cases, the initial starting designs feature a completely solid structural domain; the solid structural material in all problems is steel with a Young's modulus of $206 \mathrm{GPa}$ and shear modulus of $79.2 \mathrm{GPa}$ and a mass density of $7,800 \mathrm{~kg} \mathrm{~m}^{-3}$. The powerlaw mixing rule with $p=4$ is used in all computations to achieve material layouts that, in the end, are more or less discrete and interpretable. In addition, the nodal design variable formulation of the "Problem Formulations" Section is employed without any spatial filtering of design variables and without any perimeter control. All design optimization problems were solved using fairly standard sequential linear programming techniques with variable move limits. Optimization problems were terminated when the designs satisfied the KuhnTucker first order optimality condition.

\section{Canyon Bridge Problem}

In this case, we consider seeking optimal forms of a bridge to carry self-weight and traffic loads across a span of 1,000 m. The bridge is designed in two dimensions and the design traffic load applied uniformly to the deck level of the bridge is $10 \mathrm{kPa}$. The candidate spatial region that the bridge superstructure can potentially occupy is shown in Fig. 3(a). Since it is desired that the structural form obtained be sparse, the volume of structural material used is constrained to be less than or equal to $12.5 \%$ of the bridge envelope volume. Even with this material usage constraint, the gross weight of the bridge structure greatly exceeds the magnitude of the design traffic load. Accordingly, the bridge form is designed considering only the traffic loading, although once the designs are obtained, the performance of the structure under both traffic and self-weight loading are considered. If the self-weight loading of the bridge were considered during the optimization process, they would be dominant and unsatisfactory concept design would be obtained (see Swan et al. 1998 for both an example and discussion).

The concept design solution obtained by minimizing the linear elastic structural compliance under the design loading, without consideration of potential buckling instabilities, is shown in Fig. 3(b). The primary structural system is a long compression arch that spans the full canyon width. The deck is very slender, and supported in the first and last thirds of the span by a system of very slender compression members, and in the central third by slender tension members. The first buckling mode associated with this material layout is shown in Fig. 3(c) and indicates buckling in two of the slender compression members.

Two alternative structural concepts were obtained by solving slight variations of the optimization problem to maximize the minimum critical buckling factor subject to material usage constraints. In the first design [Fig. 3(d)], the entire structural domain $\Omega_{s}$ was treated as designable, while in the second [Fig. 3(f)] 
Table 2. Buckling Factors Computed Using Algorithm of Box 2, Along with Accurate Design Sensitivity Analysis Results Confirmed with Converged Finite Difference DSA

\begin{tabular}{|c|c|c|c|c|c|}
\hline $\begin{array}{l}f^{\text {ref }} \\
(\mathrm{N})\end{array}$ & $k$ & $\gamma$ & $\lambda^{\text {ref }}$ & $\begin{array}{l}\text { Analytical } \\
\text { DSA } \\
\text { Node } 4\end{array}$ & $\begin{array}{c}\text { Finite difference } \\
\text { DSA } \\
\text { Node } 4\end{array}$ \\
\hline 1 & $1 \times 10^{6}$ & 0.8072 & $5.1855 \times 10^{6}$ & $-7.1877 \times 10^{-7}$ & $-7.1877 \times 10^{-7}$ \\
\hline $10^{2}$ & $1 \times 10^{4}$ & 0.8072 & $5.1855 \times 10^{4}$ & $-7.1877 \times 10^{-7}$ & $-7.1877 \times 10^{-7}$ \\
\hline $10^{4}$ & $1 \times 10^{2}$ & 0.8072 & $5.1855 \times 10^{2}$ & $-7.1877 \times 10^{-3}$ & $-7.1877 \times 10^{-3}$ \\
\hline $10^{6}$ & $1 \times 10^{0}$ & 0.8072 & $5.1855 \times 10^{0}$ & $-7.1877 \times 10^{-1}$ & $-7.1877 \times 10^{-1}$ \\
\hline $10^{8}$ & $3.125 \times 10^{-2}$ & 0.3974 & $5.1855 \times 10^{-2}$ & $-7.1877 \times 10^{1}$ & $-7.1877 \times 10^{1}$ \\
\hline $10^{10}$ & $3.0517 \times 10^{-4}$ & 0.4115 & $5.1855 \times 10^{-4}$ & $-7.1877 \times 10^{3}$ & $-7.1877 \times 10^{3}$ \\
\hline $10^{12}$ & $3.8147 \times 10^{-6}$ & 0.2644 & $5.1855 \times 10^{-6}$ & $-7.1877 \times 10^{5}$ & $-7.1877 \times 10^{5}$ \\
\hline
\end{tabular}

design variable values of unity were imposed on all nodes at the deck level. The two structural concepts obtained are markedly different. The first layout resembles the compliance minimizing design [Fig. 3(b)], in that it primary feature is stout compression arch that crosses the span. The deck is substantially thicker that of the compliance minimizing design, however, reducing the need for the system of slender compression members that transfer deck loads to the arch. For this reason the secondary compression members appear to be substantially more stout than those in the compliance minimizing design. The computed fundamental buckling mode associated with this material layout [Fig. 3(e)] is not visible on the global scale and is thus a highly localized mode. In the second buckling stability design, the primary structural feature is a long deep tension member crossing the span, with a system of secondary compression members to support the deck. Since the deck would appear to be predominantly in compression along the span direction, the proposed design method has stabilized it with an irregular system of reinforcing members. The fundamental

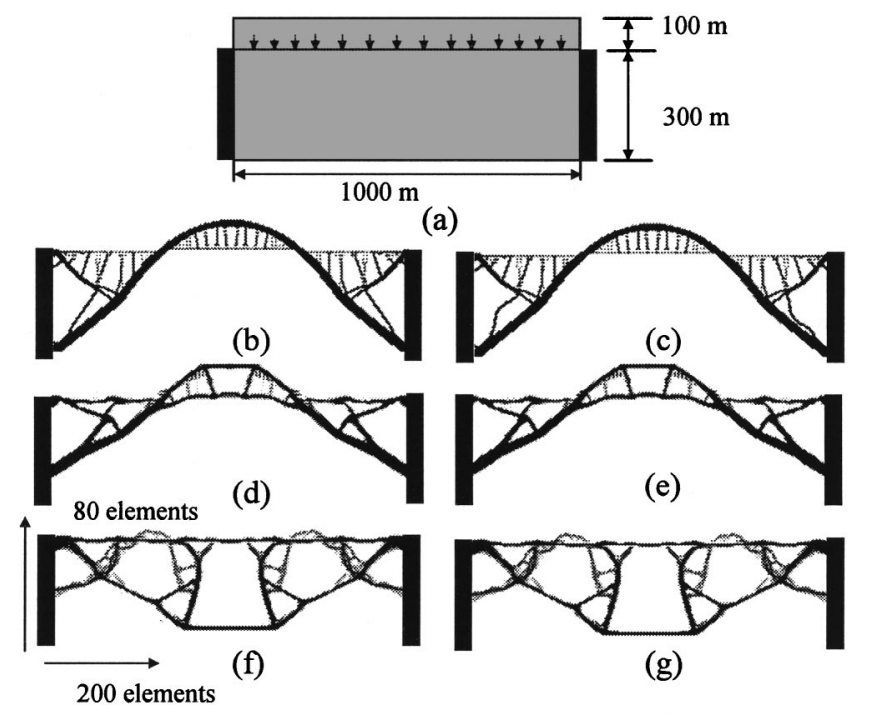

Fig. 3. (a) Design domain and boundary/loading conditions; (b) resulting material layout to minimize mean compliance of structure with lowest buckling mode (c); (d) layout to maximize minimum buckling eigenvalue with lowest buckling mode (e); (f) layout to maximize minimum buckling eigenvalue, by considering nondesignable layer along bridge deck where traction forces are applied with lowest buckling mode $(\mathrm{g})$; computed performance characteristics are provided in Table 3 buckling mode for this material layout is also highly localized and not visible on the global scale [Fig. $3(\mathrm{~g})$ ].

The computed performance characteristics associated with the three layout designs of Fig. 3 are provided in Table 3. Not surprisingly, the compliance minimizing material layout has the smallest compliance of all three designs under the traffic loading. It also shows a computed buckling eigenvalue slightly greater than that of the first buckling-resistant design [Fig. 3(d)], although substantially smaller than that of the second buckling-resistant design [Fig. 3(f)]. Under loadings that also include the self-weight of the structural material, the same trends in compliance and buckling stability persist. The computed performance characteristics of the layout designs results should be viewed cautiously, however, since in taking these concepts to more detailed final design stages, the performance characteristics could change considerably.

\section{Long-Span Concept Designs}

For main span lengths greater than 1,000 m, suspension bridges that use primarily tension to carry both the design loads and their own weight are generally optimal in that the primary structural elements are not subject to buckling. Here the conceptual layout optimization of a very long span $(3,000 \mathrm{~m})$ bridge is considered in which the candidate structural region is selected to lie at or above the traffic deck level as shown in Fig. 4(a). Again, the design traffic loading on the bridge is $10 \mathrm{kPa}$ uniformly distributed on the deck level. The structural material usage is limited to $12.5 \%$ of the envelope volume. The design domain is meshed with 10,000 bilinear continuum finite elements, and the problem is solved first to maximize the minimum critical buckling load [Fig. 4(b)] and then to minimize the structural compliance of the structure under the traffic loading [Figs. 4(c and d)]. Since the compliance-minimizing design shown in Fig. 4(c) is somewhat difficult to interpret, the problem was re solved at a substantially higher mesh resolution, and is shown in Fig. 4(d).

In both of the compliance-minimizing designs, the proposed methodology yields designs that use flexure of a flying beam-like structure whose supports are cantilevered out into the span. While these designs are in many ways quite plausible and realistic, particularly regarding the distributed support of the deck by systems of cables suspended from the compression cord of the beam, the very serious problem with these designs is that the top chord of the beam-like structure is very long, slender, and in compression under the design loading. If these compliance-minimizing concept designs were to be taken into a secondary more detailed design stage, the long compression cord members would need to be sized very large to avoid buckling, and the resulting design would be 
Table 3. Computed Performance Characteristics Associated with Three Canyon Bridge Designs of Fig. 3

\begin{tabular}{lccc}
\hline Performance measure & $\begin{array}{c}\text { Structural compliance } \\
\text { under traffic loading }(\mathrm{N} \mathrm{m})\end{array}$ & $\begin{array}{c}\text { Buckling factor } \\
\text { under traffic load only }\end{array}$ & $\begin{array}{c}\text { Buckling factor } \\
\text { under traffic load } \\
\text { and reduced self } \\
\text { weight }\end{array}$ \\
\hline Compliance design & $1.70 \times 10^{3}$ & $1.74 \times 10^{4}$ & $\begin{array}{c}\text { Bnder traffic load } \\
\text { and full self weight }\end{array}$ \\
Buckling design No. 1 & $2.49 \times 10^{3}$ & $1.47 \times 10^{4}$ & 65.9 \\
Buckling design No. 2 & $1.00 \times 10^{4}$ & $4.46 \times 10^{4}$ & 45.4 \\
\hline
\end{tabular}

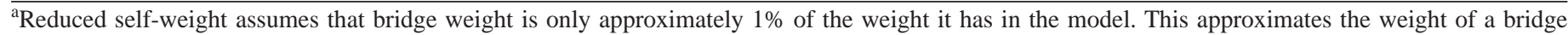
that occupies only $0.125 \%$ of the envelope volume $\Omega_{s}$.

excessively heavy and inefficient. It is worth noting that by utilizing this linear elastic compliance minimizing formulation, similar topologies will result regardless the length of the span or the magnitude of the external loads. On the other hand, the layout design of Fig. 4(b) shows a conceptual design that maximizes the linearized critical buckling load factor. As can be seen, the suspension concept design solution uses compression in the relatively stout "towers" that elevate the cable, tension in the long suspension cable that extends across the span, and tension in the relatively short hanger system that suspends the deck from the suspension cable. That the proposed formulation produces a suspension type concept design [Fig. 4(b)] resembling actual long span bridges in usage today is an encouraging development. It is worth noting here that a concept design similar to that in Fig. 4(b) was obtained by Oberndorfer et al. (1996) with a discrete ground structure topology optimization method that considered only local buckling instabilities.

To investigate the ability of the linearized buckling approach to obtain practical long-span designs under different support conditions, a similar problem but with two spans and three support
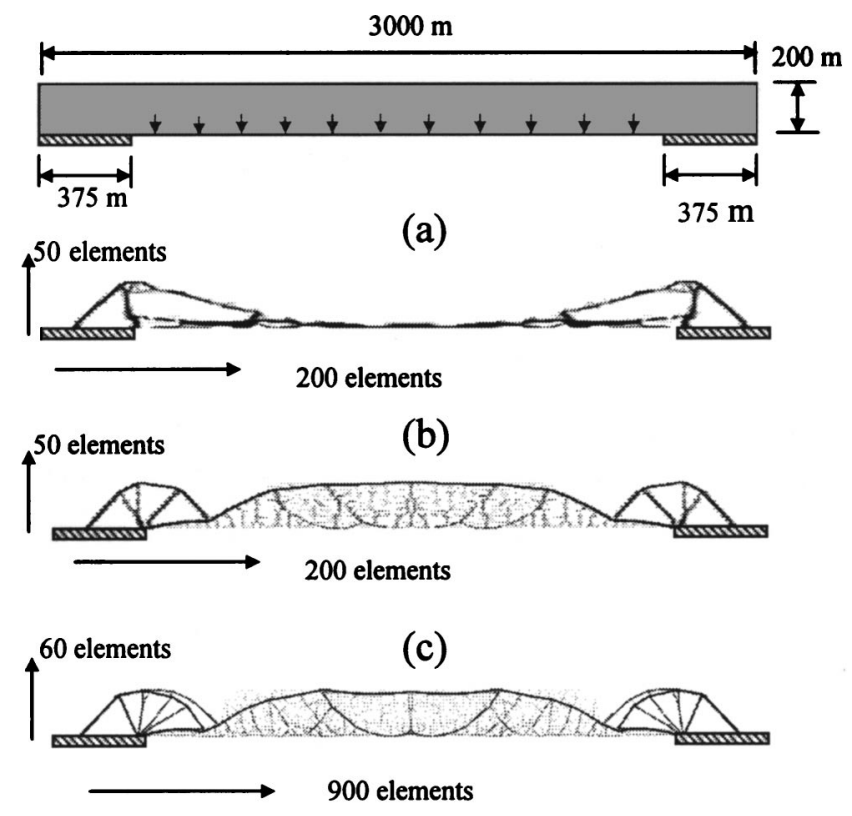

(d)

Fig. 4. (a) Design domain with loading and support conditions; (b) layout design obtained by maximizing minimum buckling eigenvalue; (c) layout design obtained by minimizing structural compliance; (d) compliance minimizing layout obtained with more refined model. Computed performance characteristics are provided in Table 4 regions [Fig. 5(a)] is solved. The layout design obtained by maximizing the buckling stability [Fig. 5(b)] again appears superior to the compliance-minimizing design solution [Fig. 5(c)] in terms of global stability.

For the long-span bridge designs, the weight of the structural material used $(12.5 \%$ of envelope volume multiplied by the unit weight of the structural material) again dwarfed the magnitude of the design traffic loading. Consequently, the weight of the structural material was neglected in the design optimization problem. The compliances and the critical buckling load factors for all of the designs in Figs. 4 and 5 are provided (Tables 4 and 5), both under the design traffic loading, and under the combined traffic loading and the weight of the structural material. Based on the computed performance characteristics shown in these tables, it appears that the layout designs obtained by maximizing the linearized buckling stability buckling factors [Figs. 4(b) and 5(b)] are indeed superior performance to the compliance-minimizing designs [Figs. 4(c), 4(d), and 5(c)] in terms of stability. As noted above, however, the only computed design performance characteristics that are truly meaningful are those based on the final detailed structural design. For long-span suspension bridges, it is actually more realistic that structural volume will lie between 0.1 and $1 \%$ of the envelope volume of the bridge. Hence, in proceeding from concept designs to realistic final detailed designs, the structural models would need to undergo substantial refinement that would significantly further reduce overall weights.
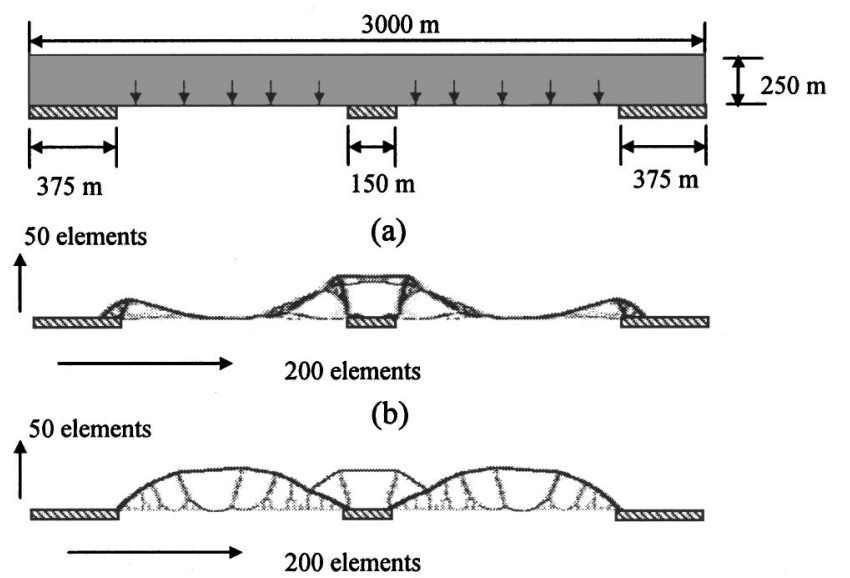

(c)

Fig. 5. (a) Design domain and boundary conditions for two-span three-support problem with all dimensions in meters; (b) resulting material layout to maximize minimal buckling eigenvalue; and (c) layout to minimize mean compliance of structure 
Table 4. Computed Performance Characteristics Associated with Long-Span Bridge Layouts Shown in Fig. 4

\begin{tabular}{lccc}
\hline & $\begin{array}{c}\text { Structural compliance } \\
\text { under traffic loading } \\
(\mathrm{N} \mathrm{m})\end{array}$ & $\begin{array}{c}\text { Buckling factor } \\
\text { under traffic load only }\end{array}$ & $\begin{array}{c}\text { Buckling factor } \\
\text { under traffic load } \\
\text { and total self weight }\end{array} \quad \begin{array}{c}\text { Buckling factor } \\
\text { under traffic load } \\
\text { and reduced self weight }\end{array}$ \\
\hline Buckling design & $3.16 \times 10^{6}$ & $1.25 \times 10^{3}$ & 5.82 \\
Compliance design & $2.92 \times 10^{5}$ & $1.68 \times 10^{2}$ & 0.882 \\
\hline
\end{tabular}

${ }^{\mathrm{a}}$ Reduced self-weight assumes that bridge weight is only approximately $1 \%$ of the weight it has in the model. This approximates the weight of a bridge that occupies only $0.125 \%$ of the envelope volume $\Omega_{s}$.

\section{Transmission Tower Design}

Failure of electrical power transmission towers by buckling during ice storms is a potential problem in the power industry. In this example, the proposed design formulation is used to obtain optimal forms in two dimensions for transmission tower that can carry the static, vertical loads associated with six cables. The static loading and boundary conditions on the spatial design domain are shown in Fig. 6(a) and are consistent with those used by Kocer and Arora (2002) in a work entailing detailed optimal design of transmission towers with respect to cross-section selection of individual members. In the current layout optimization problem with continuum topology design, the spatial domain of Fig. 6(a) is discretized with 12,000 bilinear continuum finite elements and concentrated loads of $10 \mathrm{kN}$ are applied at the six cable support positions. As in the preceding problems the structural material usage is constrained to $12.5 \%$ of the design domain's envelope volume. The design problem was first solved to minimize the mean structural compliance under the design loads, with the resulting topology shown in Fig. 6(b). While this design has a low linear elastic compliance, it is relatively unstable with regard to buckling because it utilizes many long slender unbraced compression members. As in the preceding bridge design problems, the major weakness of the compliance minimizing formulation is its inability to detect potential buckling instabilities and to arrange the structure in a way that minimizes the likelihood of their actual occurrence. Fig. 6(c) shows a layout design solution obtained by maximizing the fundamental linearized buckling eigenvalue. This solution more closely resembles the form of existing transmission towers, than the solution of Fig. 6(b). The comparative performance characteristics of both designs in terms of compliance and buckling stability are provided in Table 6 .

\section{Discussion and Conclusions}

The objective of this paper has been to present and apply a continuum structural topology optimization formulation that can be used to detect and avoid buckling instabilities in the conceptual design stage of large sparse structural systems. The motivation is to develop design tools that will produce conceptual structural forms that are optimal and that will be less problematic in the ensuing detailed design stages. Usage of the proposed formulation to maximize the minimum critical buckling load of the structure has been demonstrated here on the concept design of bridge structures and a transmission tower. Based on the example problems solved, designing to maximize the minimum critical buckling load appears to be more effective at consistently achieving stable structural forms than does minimizing the generalized compliance of the structural system. The proposed methods are somewhat promising in that they yield structural concept designs in some cases that are known to be optimal for certain design problems. For example, in the design of very long-span bridges, the method yields suspension bridge type designs. If the design tools can be confirmed on a number of such design applications, then they can be used with greater confidence in new classes of design problems for which there is not necessarily any preceding experience to guide the designer.

A number of investigators have recently demonstrated that layout optimization of sparse structures can also be achieved to maximize critical buckling loads computed via geometrically nonlinear structural buckling analysis. For large-scale sparse civil structures, the nonlinear analysis within the models can be quite computationally expensive, and the proposed formulation presented here based on linearized buckling analysis can achieve similar results much more efficiently (see Rahmatalla and Swan 2003).

The emphasis here has been on problem formulations and the resulting structural design solutions obtained. Numerical and computational issues have also been addressed to facilitate accurate calculation of buckling eigenvalues in accordance with the linearized theory, and accurate design sensitivity analysis of these buckling eigenvalues. Nonsimple (repeated) eigenvalues can and often do occur when optimizing the layout of a structure to maximize the fundamental buckling eigenvalue. While there remain unresolved issues associated with the design sensitivity of such non-simple eigenvalues, when they occur, it is worth noting here that even when the DSA expressions for simple eigenvalues are employed in such cases, the optimization problem still tends to converge, although not monotonically, to designs that achieve optimized buckling stability. The computational cost of performing the design examples of two-dimensional structures presented herein has been quite modest, being on the order of a cpu hour each on an HP J-class single processor workstation. Beyond computational resource issues, which are significant, there is no con-

Table 5. Computed Performance Characteristics of Two-Span, Three-Support, Long-Span Bridge Layouts Shown in Fig. 5

\begin{tabular}{|c|c|c|c|c|}
\hline Performance measure & $\begin{array}{l}\text { Structural compliance } \\
\text { for traffic loading } \\
(\mathrm{N} \mathrm{m})\end{array}$ & $\begin{array}{l}\text { Buckling factor } \\
\text { for traffic load only }\end{array}$ & $\begin{array}{l}\text { Buckling factor } \\
\text { for traffic load } \\
\text { and total self weight }\end{array}$ & $\begin{array}{l}\text { Buckling factor } \\
\text { for traffic load and } \\
\text { reduced self weight }{ }^{\mathrm{a}}\end{array}$ \\
\hline Buckling design & $1.52 \times 10^{5}$ & $4.84 \times 10^{3}$ & 63.3 & $5.15 \times 10^{3}$ \\
\hline Compliance design & $2.06 \times 10^{4}$ & $1.94 \times 10^{3}$ & 7.18 & $5.29 \times 10^{2}$ \\
\hline
\end{tabular}

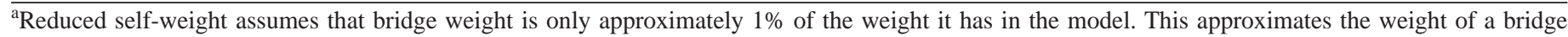
that occupies only $0.125 \%$ of the envelope volume $\Omega_{s}$. 


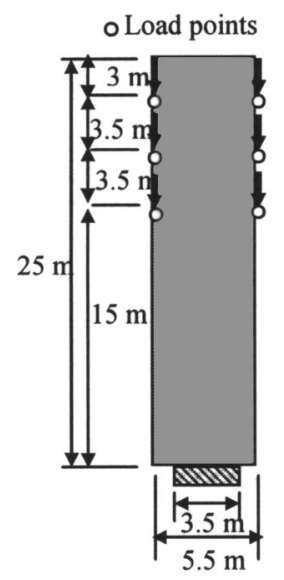

(a)

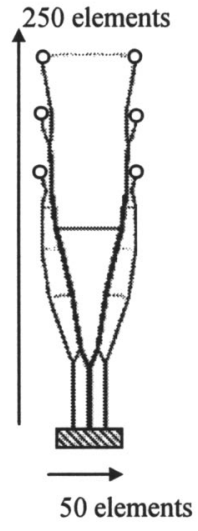

(b)

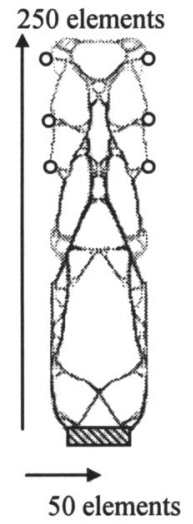

(c)
Fig. 6. (a) The design domain and boundary/loading conditions for the transmission tower; (b) resulting layout for compliance minimization; (c) resulting layout to optimize global stability. Computed performance characteristics are in Table 6

Table 6. Computed Performance Characteristics Associated with Transmission Tower Layout Designs Shown in Fig. 6

\begin{tabular}{lcc}
\hline Performance measure & Compliance $(\mathrm{N} \mathrm{m})$ & Buckling eigenvalue \\
\hline Buckling design & 25.0 & $2.44 \times 10^{3}$ \\
Compliance design & 0.782 & $3.37 \times 10^{2}$ \\
\hline
\end{tabular}

ceptual difficulty in extending the proposed design methods to form-finding of structures in three dimensions.

In the example problems solved in "Demonstrative Examples," the structure was permitted to occupy up to $12.5 \%$ of the structure's envelope volume. In reality, suspension bridges and transmission towers typically feature a higher degree of sparsity, occupying on the order of $1 \%$ or less of the structure's envelope volume. Within the current continuum topology optimization framework, modeling structures with such a high degree of sparsity would require finite element models of much greater resolution than the models used herein with $10^{4}-10^{5}$ elements. Nevertheless, even though the current stability optimization framework is limited in its ability to capture the true sparsity characteristics of structures, it does yield material layouts that are quite realistic.

\section{Acknowledgments}

This research was funded in part by a grant from the University of Iowa CIFRE Program, and in part by Grant No. NSF DMS9874015.

\section{References}

Achtziger, W. (1999). "Local stability of trusses in the context of topology optimization Part II: A numerical approach." Struct. Optim., 17, 247-258.

Bathe, K. J. (1996). Finite element procedures, Prentice-Hall, Englewood Cliffs, N.J.

Bendsøe, M. P., and Kikuchi, N. (1988). "Generating optimal topology in structural design using a homogenization method." Comput. Methods Appl. Mech. Eng., 71, 197-224.
Bendsøe, M. P., and Sigmund, O. (1999). "Material interpolations in topology optimization.” Arch. Appl. Mech., 69, 635-654.

Bojczuk, D., and Mróz, Z. (1999). "Optimal topology and configuration design of trusses with stress and buckling constraints." Struct. Optim., $17,25-35$.

Bruns, T. E., and Tortorelli, D. A. (2001). "Topology optimization of nonlinear elastic structures and compliant mechanisms." Comput. Methods Appl. Mech. Eng., 190, 3443-3459.

Buhl, T., Pedersen, W., and Sigmund, O. (2000). "Stiffness design of geometrically nonlinear structures using topology optimization." Struct. Multidisc. Optim., 19, 93-104.

Cheng, K. T., and Olhoff, N. (1981). "An investigation concerning optimal design of solid elastic plates." Int. J. Solids Struct., 17, 305-323.

Choi, K. K., Haug, E. J., and Seong, H. G. (1983). "An iterative method for finite dimensional structural optimization problems with repeated eigenvalues." Int. J. Numer. Methods Eng., 19, 93-112.

Dobbs, W., and Felton, L. P. (1969). "Optimization of truss geometry." Proc. ASCE, 95(ST10), 2105-2519.

Dorn, W., Gomory, R., and Greenberg, H. (1964). "Automatic design of optimal structures." J. Mech., 3, 25-52.

Eschenauer, H. A., and Olhoff, N. (2001). "Topology optimization of continuum structures: A review." Appl. Mech. Rev., 54, 331-390.

Gea, H. C., and Luo, J. (2001). "Topology optimization of structures with geometrical nonlinearities." Comput. Struct., 79, 1977-1985.

Guedes, J. M., and Rodrigues, H. C. (1995). "Necessary conditions for the optimal design of structures with nonsmooth eigenvalue based criterion." Struct. Optim., 9, 52-56.

Hemp, W. S. (1973). Optimum structures, Clarendon, Oxford, U.K.

Kocer, F. Y., and Arora, J. S. (2002). "Optimal design of latticed towers subjected to earthquake loading." J. Struct. Eng., 128(2), 197-204.

Kočvara, M. (2002). "On the modeling and solving of the truss design problem with global stability constraints." Struct. Multidisc. Optim., 23, 189-203.

Ma, Z. D., Kikuchi, N., and Cheng, H. C. (1995). “Topological design for vibrating structures." Comput. Methods Appl. Mech. Eng., 121, 259280.

Michell, A. G. M. (1904). "The limits of economy in frame structures." Philos. Mag., 8(47), 589-597.

Mijar, A. R., Swan, C. C., Arora, J. S., and Kosaka, I. (1998). "Continuum topology optimization for concept design of frame bracing systems.” J. Struct. Eng., 124(5), 541-550.

Min, S., Nishiwaki, S., and Kikuchi, N. (2000). "Unified topology design of static and vibrating structures using multiobjective optimization." Comput. Struct., 75, 93-116.

Neves, M. M., Rodrigues, H., and Guedes, J. M. (1995). “General topology design of structures with a buckling load criterion." Struct. Optim., 10, 71-78.

Oberndorfer, J. M., Achtziger, W., and Hörnlein, H. R. E. M. (1996). "Two approaches for truss topology optimization: a comparison for practical use." Struct. Optim., 11, 137-144.

Ohsaki, M., and Swan, C. C. (2002). "Topology and geometry optimization of trusses and frames." ASCE/SEI State-of-Art-Rep. on Structural Optimization, S. A. Burns, ed., New York.

Rahmatalla, S. F., and Swan, C. C. (2003). "Continuum topology optimization of buckling-sensitive structures." AIAA J., 41(6), 11801189.

Rozvany, G. I. N. (1976). Optimal design of flexural systems, Pergamon, Oxford, U.K.

Rozvany, G. I. N. (1996). "Difficulties in truss topology optimization with stress, local buckling and system stability constraints." Struct. Optim., 11, 213-217.

Save, M., and Prager, W. (1990). Structural optimization, Plenum, New York.

Seyranian, A. P., Lund, E., and Olhoff, N. (1994). "Multiple eigenvalues in structural optimization problems." Struct. Optim., 8, 207-227.

Swan, C. C., and Arora, J. S. (1997). “Topology design of material layout 
in structured composites of high stiffness and strength." Struct. Optim., 13(1), 45-59.

Swan, C. C., Arora, J. S., Kosaka, I., and Mijar, A. R. (1998). "Concept design of bridge structures for stiffness and vibrations using continuum topology optimization." Structural engineering worldwide, N. K. Srivastava, ed., ASCE, New York.

Swan, C. C., and Kosaka, I. (1997). "Voigt-Reuss topology optimization for structures with nonlinear material behaviors." Int. J. Numer. Methods Eng., 40, 3785-3814.

Yin, L., and Ananthasuresh, G. K. (2002). "A novel topology design scheme for multiphysics problems of electro-thermally actuated compliant micromechanisms." Sens. Actuators A, 3226, 1-12.

Zou, M. (1996). "Difficulties in truss topology optimization with stress and local buckling constraints." Struct. Optim., 11, 134-136. 